\title{
A rare cause of acute abdominal pain in a patient with Primary ciliary dyskinesia with situs inversus totalis
}

\author{
Kısmet Çık1 ${ }^{1 \oplus}$, Özlem Boybeyi Türer ${ }^{2}$, Mina Hızal $^{3}$, Gökçen Dilşa Tuğcu ${ }^{3}$, \\ Nagehan Emiralioğlư ${ }^{3}$ Ebru Yalçın³, Deniz Doğru Ersöz ${ }^{3}$, Nural Kiper³, \\ Uğur Özçelik ${ }^{3}$
}

Departments of ${ }^{1}$ Pediatrics, ${ }^{2}$ Pediatric Surgery and ${ }^{3}$ Pediatric Pulmonology, Hacettepe University Faculty of Medicine, Ankara, Turkey.

\begin{abstract}
Primary ciliary dyskinesia (PCD) is a rare, genetic disease characterized by ciliary dysfunction. Patients may present with respiratory distress during neonatal period; chronic sinopulmonary disease, bronchiectasis, recurrent otitis media, sinusitis and infertility in later periods. About 50\% of PCD patients have situs inversus totalis and $6-12 \%$ have situs ambiguous known as heterotaxy syndromes. Herein, we present a case of PCD and accompanying situs inversus who had acute abdominal pain and was diagnosed with torsion of one of the multiple spleens. Evaluation of acute abdominal pain in these patients has great importance since the internal organs are not at their typical locations.
\end{abstract}

Key words: Kartagener syndrome, primary ciliary dyskinesia, spleen torsion.

Primary ciliary dyskinesia(PCD) is a rare, genetic disease characterized by ciliary dysfunction. Patients may present with respiratory distress during neonatal period; chronic sinopulmonary disease, bronchiectasis, recurrent otitis media, sinusitis and infertility in later periods. About $50 \%$ of PCD patients have situs inversus totalis and $6-12 \%$ have situs ambiguous known as heterotaxy syndromes. ${ }^{1,2}$ Situs inversus is a condition in which the arrangement of the internal organs is a mirror image of normal anatomy. In heterotaxy syndrome internal organs arrange non-mirror image, abnormal, and in mixed location. Cardiac, large vessel and lung anomalies, polysplenia and asplenia can be seen in situs ambiguous.

Therefore, evaluation of acute abdominal pain in these patients has great importance since

\section{Kismet Çık1 \\ dr.ksmet@hotmail.com}

Received 15th December 2018; revised 10th January 2019; accepted 10th January 2019

This study was presented at the 20th Turkish Thorax Congress, 5-9 April 2017, Antalya, Belek, Turkey. the internal organs are not at their typical locations. Herein, we present a case of PCD and accompanying situs inversus who had acute abdominal pain and was diagnosed with torsion of one of the multiple spleens.

\section{Case Report}

A 15-year-old male patient was referred to our hospital for acute abdominal pain with the initial diagnosis of acute appendicitis. $\mathrm{He}$ has been followed at our hospital with the diagnosis of PCD for two years. He presented with an abdominal pain that was more prominent in lower right regions. There was no fever, vomiting, diarrhea, constipation, urinary frequency or dysuria. On physical examination he had tenderness on palpation, defense and rebound tenderness at the lower right. There was no swelling, tenderness and pain in testicular examination. There were rales in the lung bases. Leukocytosis was found in blood count with $72,5 \%$ neutrophil predominance, other laboratory tests were within normal limits. Oral feeding was discontinued because 
of the acute abdomen. Intravenous fluid support was started. Abdominal ultrasonography was performed with the preliminary diagnosis of acute appendicitis. It was found that the liver was located on the left side, the spleen was located on the right side and there were six accessory spleens around the spleen. There was no vascularization in the largest accessory spleen $(5 \times 6 \times 6 \mathrm{~cm})$. An emergent surgery was performed since he had acute abdominal signs and it was suspected that there was torsion of one of the multiple spleens. Peroperatively it was seen that there were five accessory spleens and the largest one was swollen, edematous and rotated five times on itself (Fig. 1). Torsed spleen and all accessory spleens were excised. The original spleen and its ligaments were normal and not excised. Appendectomy was also performed since he had situs inversus totalis. On pathological examination, inflammation and congestion was seen in torsed accessory spleen. On the fourth day after the operation, the patient was discharged with cure. The patient is still under follow-up uneventfully.

Informed consent was received from the family.

\section{Discussion}

Primary ciliary dyskinesia is a genetic disorder characterized by motile silia dysfunction, impaired mucociliary clearance and recurrent respiratory infections. It is an autosomal recessive disorder but $\mathrm{X}$-linked inheritance pattern has rarely been reported. It affects approximately 1 in 15,000-20,000 individuals. ${ }^{3}$ The first PCD case who had bronchiectasis and situs inversus was reported by Siewart. Bronchiectasis, sinusitis and situs inversus triad was described by Kartagener in 1933 and it is known as Kartagener syndrome. ${ }^{2}$ Respiratory distress is seen in the newborn; cough, nasal congestion, nasal polyp, chronic pansinusitis, recurrent middle ear infection, hearing loss, recurrent lower respiratory tract infection, and bronchiectasis are seen in the follow up. Males are $100 \%$ infertile, whereas fertility is decreased in females. Cystic kidney, cystic-cholestatic liver, skeletal deformities, hydrocephalus, developmental retardation, retinitis pigmentosa, blindness and deafness may also occur due to non-motile ciliary dysfunction. Clinical and diagnostic tests are both used for diagnosis. Genetic tests, cilia examination in electron microscopy, cilia motility study and nasal nitric oxide are diagnostic tests. Genetic studies and expert evaluation are recommended for clinical conditions of immotile silia dysfunction in patients with PCD. ${ }^{4}$

PCD and situs inversus are both seen as a result of ciliary dysfunction. Airway epithelium cilia defects cause PCD while nodal cilia, which play an important role during embryogenesis,
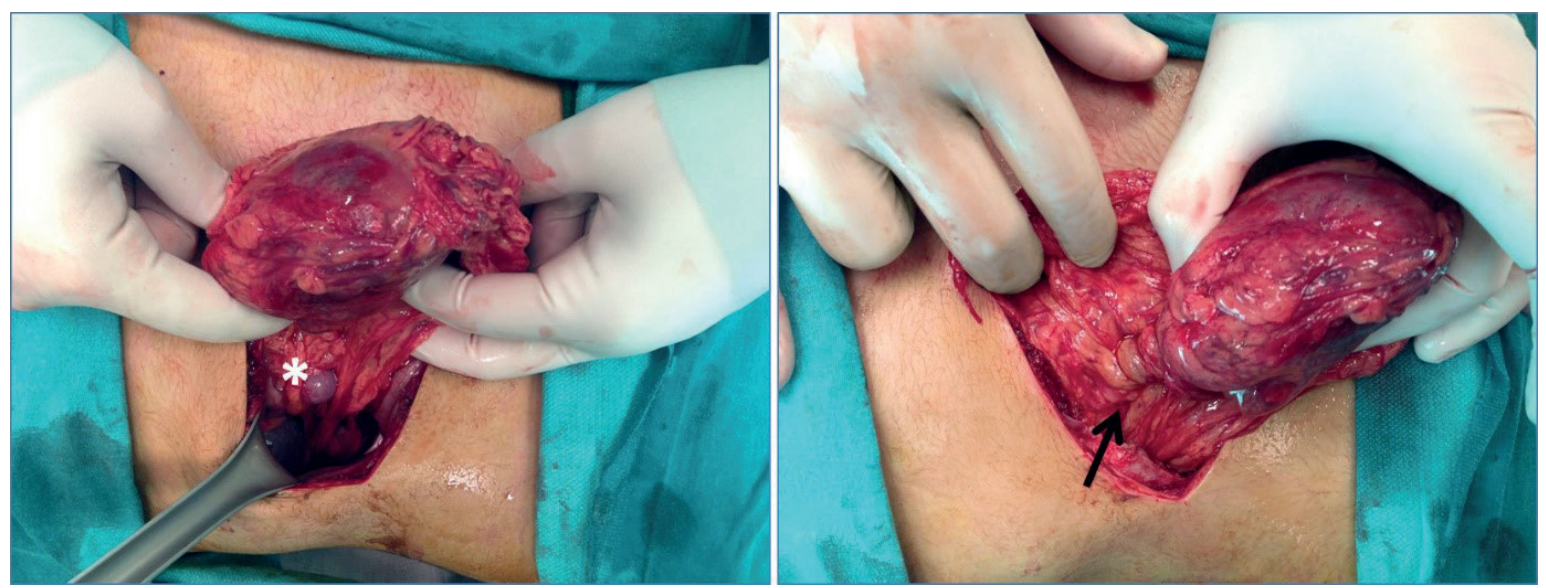

Fig. 1. The surgical view; the accessory spleens are seen in the left hand side $\left(^{*}\right)$ and the torsion of the largest accessory spleen is seen in the right hand side (torsed vascular pedicle signed with an arrow). 
defects cause situs inversus. ${ }^{5}$ Situs inversus is a condition in which the arrangement of the internal organs is a mirror image of normal anatomy. In situs ambiguous, internal organs arrange an abnormal, non-mirror image, and exist in mixed location. The prevalence of isolated situs inversus totalis is 1/8500; heterotaxy is $1 / 10000$. About $50 \%$ of PCD patients have situs inversus totalis and 6-12\% have situs ambiguous known as heterotaxy syndromes. The true prevalence may be even higher, as many PCD patients do not routinely have investigations to define their abdominal laterality defects. Although the prevalence of an approximate heterotaxy in PCD patients is known, the PCD prevalence in heterotaxy syndrome is unknown. ${ }^{6}$ Heterotaxy syndromes are divided into 2 groups according to whether it is polysplenia or asplenia. Heterotaxy syndromes with polysplenia are called left isomerism; heterotaxy syndromes with asplenia are called right isomerism. Left isomerism is characterized with partial pulmonary venous return anomalies, polysplenia, left superior vena cava, left inferior vena cava, left atrium, and bilateral two-lobe lungs while right isomerism is characterized with right ventricle, bilateral superior vena cava, total pulmonary venous return anomaly, pulmonary atresia, pulmonary stenosis, bilateral three lobed lungs and asplenia. Different locations of the organs, biliary atresia, choledocholithiasis, annular pancreas, short pancreas, pancreatitis due to short pancreas, malrotation, obstruction, volvulus, inferior vena cava and portal venous system anomalies are gastrointestinal complications of heterotaxis syndromes. Vascular anomalies may cause bleeding and thrombosis in abdominal surgeries. ${ }^{7}$

Location and number of spleens are variable in heterotaxy syndromes with polysplenia. Although they are usually asymptomatic, they can mimic lymphadenopathy or a tumor. It can also cause symptoms due to torsion, haemorrhage, spontaneous rupture or cyst formation. ${ }^{8}$ The torsion of spleen at polysplenia is a very rare condition causing acute abdominal pain and has also been reported in a few reports in the literature. Patients present with pain, vomiting and nausea in the splenic torsion. It has been reported that the pain may mimic acute appendicitis. ${ }^{9}$ Our patient's pain was more prominent in the right lower torso and we initially considered as acute appendicitis. Splenic torsion is rarely diagnosed preoperatively because it is an extremely rare entity. Imaging methods are used to diagnose and some cases need laporotomy for an exact diagnosis. The excision of torsed spleen is the prompt treatment. ${ }^{9}$

The coincidence of PCD with this rare condition is also very rare. There were some reported cases in literature of splenic torsion in heterotaxy syndromes with polysplenia and first case was reported by Ackerman in $1982 .{ }^{10}$ Splenic torsion is reported in heterotaxy syndromes with polysplenia in the literature. There was no information as to whether PCD evaluation was performed in these cases. We could find only one case who had PCD and splenic torsion in the literature. ${ }^{11}$

Polysplenia should be kept in mind for patients who are followed up with PCD and situs inversus ('Kartagener Syndrome') and abdominal imaging should definitely be performed. Splenic torsion should be considered in the differential diagnosis of patients who are known to have polysplenia and admitted with the complaint of abdominal pain. Awareness of this entity is important in the exact diagnosis and prompt treatment of such cases.

\section{REFERENCES}

1. Zariwala MA, Knowles MR, Leigh MW. Primary ciliary dyskinesia, In: Pagon RA, Bird TC, Dolan CR, Stephens K (eds). GeneReviews (Internet). Seattle, WA: University of Washington, Seattle; 1993-2016. National Center for Biotechnology website. https:// www.ncbi.nlm.nih.gov/books/NBK1122/

2. Fretzayas A, Moustaki M. Clinical spectrum of primary ciliary dyskinesia in childhood. World J Clin Pediatr 2016; 5: 57-62. 
3. Popatia R, Haver K, Casey A. Primary ciliary dyskinesia: an update on new diagnostic modalities and review of the literature. Pediatr Allergy Immunol Pulmonol 2014; 27: 51-59.

4. Shapiro AJ, Zariwala MA, Ferkol T, et al; Genetic Disorders of Mucociliary Clearance Consortium. Diagnosis, monitoring, and treatment of primary ciliary dyskinesia: PCD foundation consensus recommendations based on state of the art review. Pediatr Pulmonol 2016; 51: 115-132.

5. Fliegauf M, Benzing T, Omran H. When cilia go bad: cilia defects and ciliopathies. Nat Rev Mol Cell Biol 2007; 8: 880-893.

6. Shapiro AJ, Tolleson-Rinehart S, Zariwala MA, Knowles MR, Leigh MW. The prevalence of clinical features associated with primary ciliary dyskinesia in a heterotaxy population: results of a web-based survey. Cardiol Young 2015; 25: 752-759.

7. Kim S, Lee YS, Jung JH. Anomalies of abdominal organs in polysplenia syndrome: multidetector computed tomography findings. J Korean Soc Radiol 2016; 74: 114-122.
8. Chowdhary R, Raichandani L, Kataria S, Raichandani S, Joya H, Gaur S. Accessory spleen and its significance: a case report. Int J App Res 2015; 1: 902-904.

9. Dash MR, Upasani AV, Chandna SB, Rathod PB, Prajapati KK, Patel DN. Splenic torsion in a child with polysplenia and situs inversus: a very rare presentation. Indian J Surg 2013; 75(Suppl 1): 236237.

10. Ackerman NB Jr, Smith MD, Strobel CT, Wheller JJ Splenic torsion in the polysplenia syndrome. South Med J 1982; 75: 897-898.

11. Tonello P, Carbone A. Twisting of the spleen observed in a case of polysplenic disorder associated with situs viscerum inversus and Kartagener's syndrome. Pathol Res Pract 1989; 185: 523-525. 